\title{
Intramucosal true perineurioma of the sigmoid: endoscopic and pathological features of an infrequent lesion
}

A 59-year-old woman was admitted to our hospital with complaints of recurrent non-intensive pain in the right side, constipation, and abdominal distension. The patient had a medical history of dolichocolon. Her physical examination and laboratory test results were unremarkable. Abdominal ultrasonography showed moderate diffuse parenchymal changes in the liver (liver steatosis) and pancreas. Colonoscopy revealed redundant colon with a non-epithelial lesion of the sigmoid that had a diameter of $0.6 \mathrm{~cm}$ with a smooth surface and regular capillary pattern ( $\triangleright$ Fig. 1; $>$ Video 1), which was biopsied during the procedure.

Histopathological examination of the biopsy demonstrated a proliferation of short spindle cells with pale cytoplasm and indistinct cell borders, whose nuclei were bland and not wavy. The lesion was situated in the lamina propria mucosae and lacked serrated or hyperplastic epithelial changes. Necrosis, pleomorphism, and mitotic figures were absent ( Fig.2a). Immunohistochemical staining with epithelial membrane antigen (EMA) showed weak diffuse positivity ( Fig.2 b), and with S100 showed focal positive staining ( $\vee$ Fig. $\mathbf{2 c}$ ). An intramucosal true perineurioma of the sigmoid was confirmed.

After the diagnosis had been made, the patient underwent endoscopic resection of the lesion with a cold snare. There were no complications in the postoperative period and the patient was discharged on the second day after surgery. A perineurioma is a rare benign peripheral nerve sheath tumor composed of cells resembling normal perineurium, with a reported incidence of $0.1 \%-1.46 \%$ [ 1 , 2]. Since their first description in 2004 by Eslami-Varzaneh, about 150 cases of perineurioma have been reported in the colorectum to date [1,3]. Pai et al. [4] suggested that a perineurioma is the result of a reactive process, likely induced
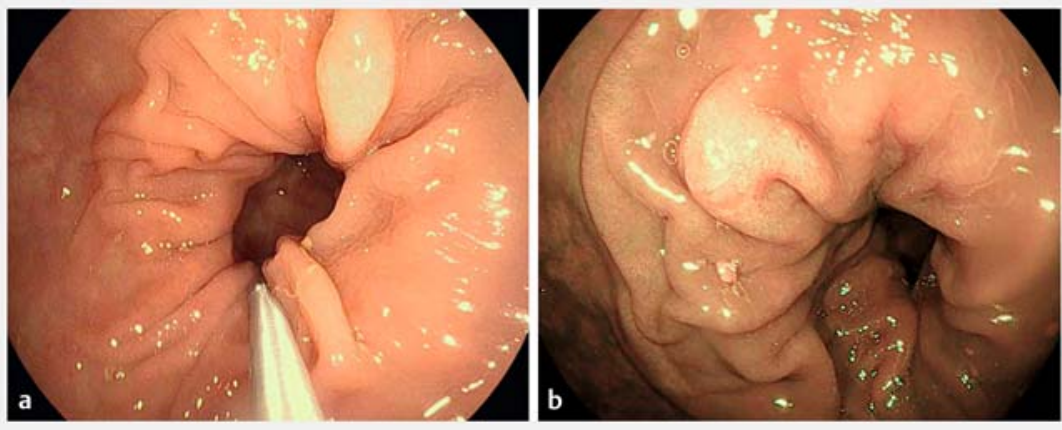

- Fig. 1 Colonoscopy images before biopsy showing a non-epithelial lesion of the sigmoid: a in white light mode; $\mathbf{b}$ in IScan mode.
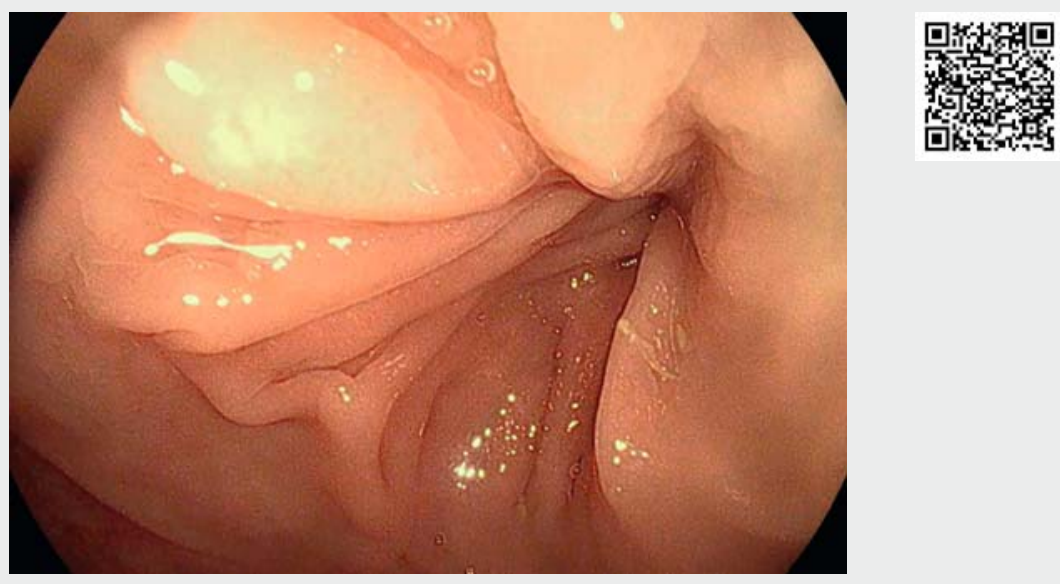

Video 1 Colonoscopy showing a non-epithelial lesion with a diameter of $0.6 \mathrm{~cm}$, a smooth surface, and regular capillary pattern in the sigmoid colon, which was biopsied.

by the BRAF-mutated serrated epithelium. In our case, serrated or hyperplastic epithelial changes were not revealed, so we suggest that we are dealing with a true perineurioma of the sigmoid. An electronic search of the PubMed database (January 2000 - January 2020) using the keywords "true perineurioma colon" revealed only two reports.

Endoscopy_UCTN_Code_CCL_1AD_2AC

\section{Competing interests}

The authors declare that they have no conflict of interest. 

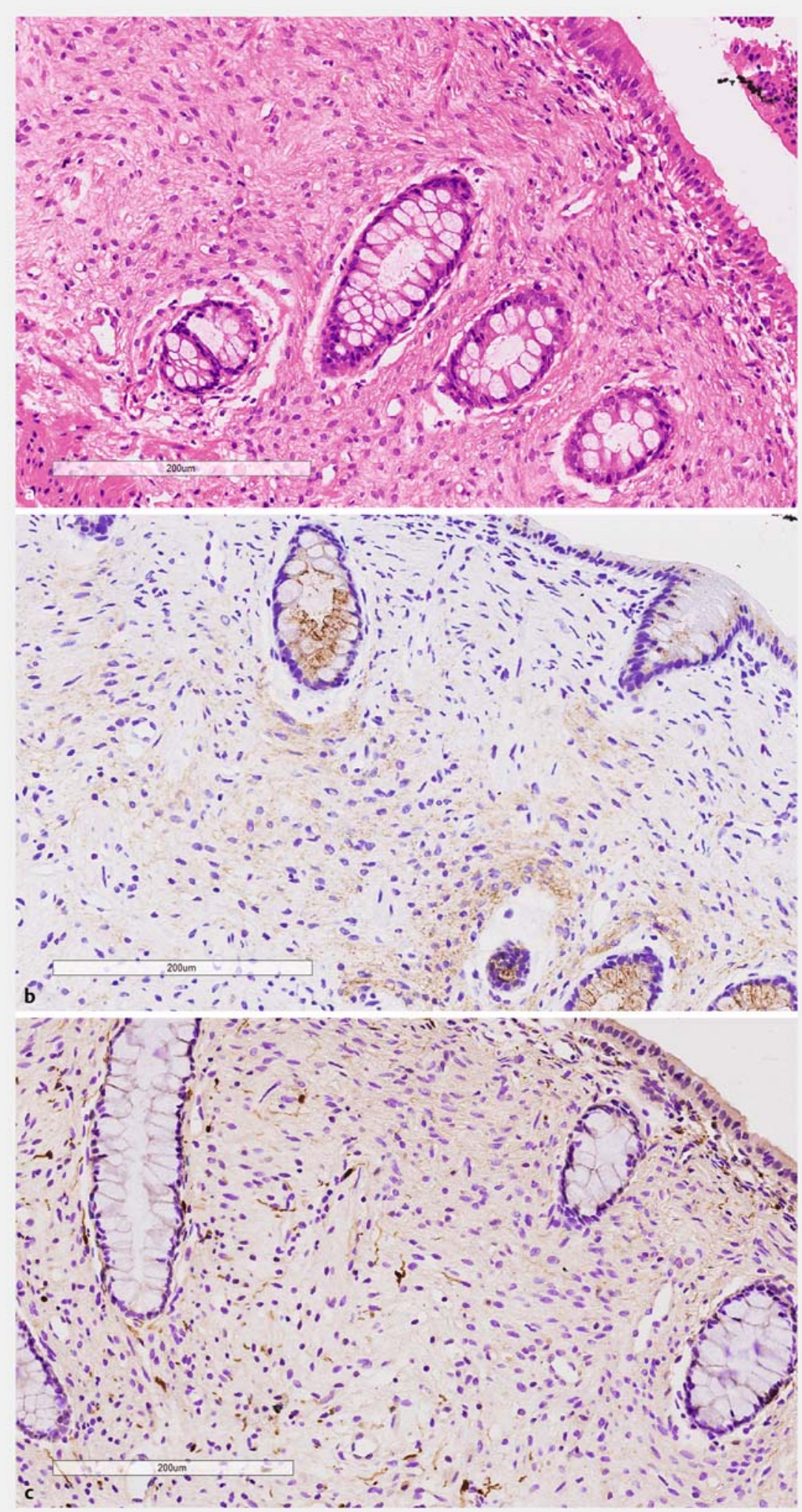

- Fig. 2 Histopathology of the biopsy showing: a a proliferation of short spindle cells with pale cytoplasm and indistinct cell borders, bland non-wavy nuclei, and lacking serrated or hyperplastic epithelial changes in the lamina propria mucosae (hematoxylin and eosin [H\&E] stain; magnification $\times 250$ ); b weak diffuse positive immunohistochemical staining with epithelial membrane antigen $(E M A$; magnification $\times 250)$; $c$ focal positive immunohistochemical staining with S100 (magnification $\times 250$ ).
The authors

Andrey P. Kiryukhin', Pavel V. Pavlov'1, Alexander A. Fedorenko ${ }^{1}$, Alexander $\mathrm{S}$. Tertychnyy ${ }^{2}$

1 Endoscopy Unit, The Second University Clinic, I. M. Sechenov First Moscow State Medical University (Sechenov University), Moscow, Russia

2 Department of Pathology, I. M. Sechenov First Moscow State Medical University (Sechenov University), Moscow, Russia

\section{Corresponding author}

\section{Andrey P. Kiryukhin, MD, PhD}

Endoscopy Unit, The Second University Clinic, I. M. Sechenov First Moscow State Medical University (Sechenov University), 1 build. 1. Pogodinskaya St, 119435 , Moscow, Russia

a.p.kiryukhin@gmail.com

\section{References}

[1] Motta F, Spadola S, Bosco A et al. Perineurioma of the colon: an uncommon tumor with an unusual location. Report of a case and review of the literature. Pathologica 2018; 110: 111-115

[2] Grech P, Schofield JB. Spindle cell proliferations of the sigmoid colon, rectum and anus: a review with emphasis on perineurioma. Histopathology 2020; 76: 342-353

[3] Eslami-Varzaneh F, Washington K, Robert ME et al. Benign fibroblastic polyps of the colon: a histologic, immunohistochemical, and ultrastructural study. Am J Surg Pathol 2004; 28: 374-378

[4] Pai RK, Mojtahed A, Rouse RV et al. Histologic and molecular analyses of colonic perineurial-like proliferations in serrated polyps: perineurial-like stromal proliferations are seen in sessile serrated adenomas. Am J Surg Pathol 2011; 35: 1373-1380

Bibliography

\section{Endoscopy 2020; 52: E394-E395}

DOI 10.1055/a-1144-2518

ISSN 0013-726X

published online 17.4 .2020

(c) 2020. Thieme. All rights reserved. Georg Thieme Verlag KG, Rüdigerstraße 14, 70469 Stuttgart, Germany 